\title{
VIBRATION WITH NON-LINEAR DAMPING. STOCHASTIC APPROACH
}

\author{
M. LAMRHARI
}

1. Laboratoire d'Etude des Matériaux Avancés et Applications, FS-EST, Moulay Ismail University, Meknès, Maroc

\begin{abstract}
:
This paper describes a stochastic approach to the vibration behavior of a nonlinear damping structure. The deterministic approach is based on motion equations including the non-linearity of damping. For the stochastic calculation, the deviations were assigned to the input parameters of the mathematical model. The probabilistic distribution of these parameters must reflect real operational situations. Numerical simulations were performed with MATLAB using the Monte Carlo and perturbation method. The resulting curves for the parameters studied can find a practical application in the optimization of rotating machines (washing machine), where the parameters of mass, position of the center of gravity, etc. change during operation.
\end{abstract}

Keywords: Nonlinear damping, stochastic, Monte Carlo, perturbation method.

\section{Introduction}

In a mechanical system, the damping force is a function of velocity, in several systems, this function is nonlinear. In the car suspension system, the non-linear damper provides an adequate model for predicting the handling quality [1]. In the flow orifices, the pressure across the orifice is proportional to the square of the velocity, which is an example of the quadratic dependence of the force on the velocity of flow [2]. $n$ the aerospace sector, structures are modeled with non-linear damping [3]. In the field of nanotechnology, nanotubes are modeled with nonlinear damping [4]. This paper describes the free vibration of a structure taking into account the influence of the non-linearity of viscous dampers. The calculations are based on the motion equations, the mathematical expression of the non-linearity of the damping is given by the characteristics of the damping elements. This paper deals with the stochastic calculation of the vibration as a function of the variance mass parameter. Stochastic calculations are execute using the Monte Carlo simulation [5] and perturbation method [6]. The resulting curves for the studied parameters can find a practical application in the optimization of the systems.

\section{Stochastic nonlinear modeling.}

\subsection{Modeling the nonlinear damping force :}

To determine the vibration curves of a system, it is necessary to specify the nonlinear damping function $f_{n l}(\dot{x})$, which best correspond the real data. The appropriate function has been specified in the following form:

$$
f_{n l}(\dot{x})=\operatorname{sign}(\dot{x}) \cdot c_{1} \cdot|\dot{x}|^{c_{2}}
$$

Where the function of sign is equal to 1 when the speed $\dot{x}(t)$ is greater than zero and equal to -1 if $\dot{x}(t)$ is less than zero.

Figure 1 shows the damping force for $c_{1}=140 \mathrm{Kg} / \mathrm{s}$ and $c_{1}=0.2$

The dynamic equation of one degree of freedom system with nonlinear damping can be written as:

$$
\mathrm{m} \ddot{\mathrm{x}}+\mathrm{c} \dot{\mathrm{x}}+f_{n l}(\dot{x})+\mathrm{kx}=\mathrm{f}(\mathrm{t})
$$

With: $\mathrm{m}, \mathrm{c}$ and $\mathrm{k}$ are respectively mass, linear damping and stiffness; $x(t)$ is the temporal response of the system and $f(t)$ is the input excitation force.

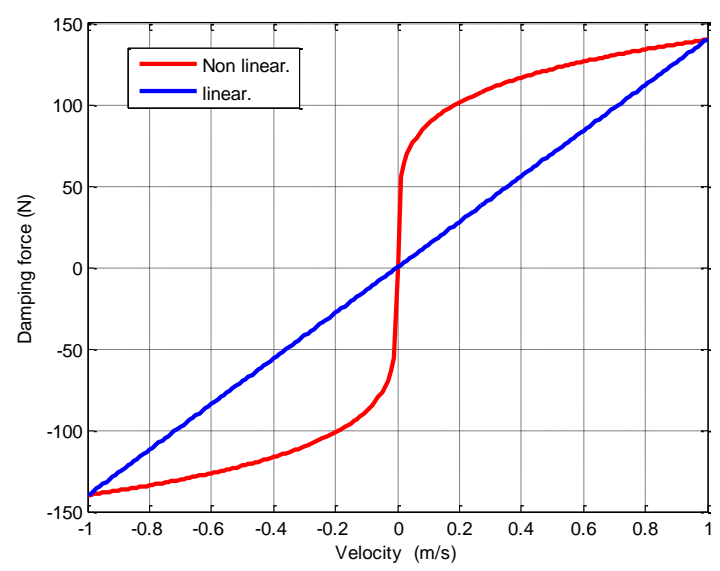

Figure 1: Linear and nonlinear damping force

\subsection{Modeling uncertainties}

During the life cycle of the structure, some parameters prove to be random, in this paper to the study is limited of two random parameters the mass $m$ and the stiffness $k$, their stochastic behavior is describes using the random variables Gaussian so these parameters are described by the following relationships:

$$
\tilde{m}=\bar{m}\left(1+\vartheta_{m} \aleph_{1}\right)
$$

$\bar{m}$ et $\vartheta_{m}$ denote the average value and the mass coefficient variation.

$$
\tilde{k}=\bar{k}\left(1+\vartheta_{k} \aleph_{2}\right)
$$


$\bar{k}$ et $\vartheta_{k}$ denote the average value and the stiffness coefficient variation.

$\aleph_{1}$ et $\aleph_{2}$ are reduced centered Gaussian random variables.

\section{Solving the stochastic nonlinear problem}

\subsection{Deterministic case:}

It is very difficult to find a solution for induced vibrations with the nonlinear damping function. Therefore the possible solution includes the linearization of the damping function. The advantage of numerical computation is the possibility of using nonlinear damping characteristics, which is the better represent of reality than the linearization damping function.

The calculation assumes a regular acceleration of the movement at very short intervals $\Delta t=0,0001 \mathrm{~s}$. The resolution is done by iteration, by the Newmark method with an initial displacement $x_{0}=0.03(\mathrm{~m})$ and an initial velocity $\dot{x}_{0}=0(\mathrm{~m} / \mathrm{s})$.

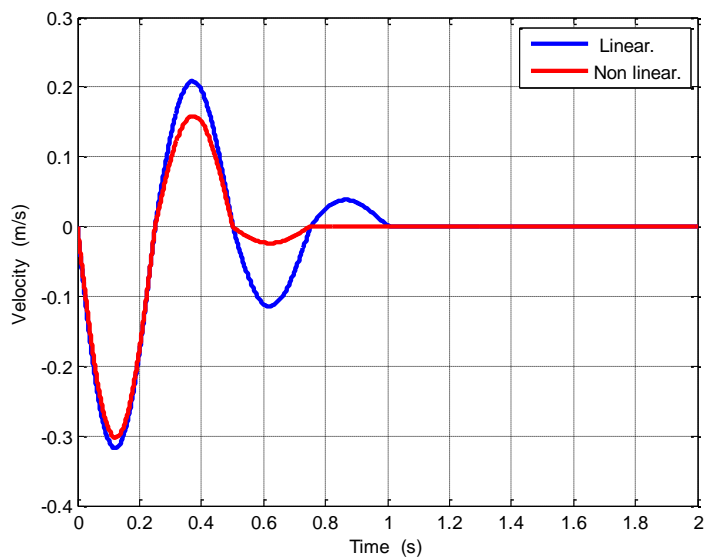

Figure2: Linear and nonlinear velocity.

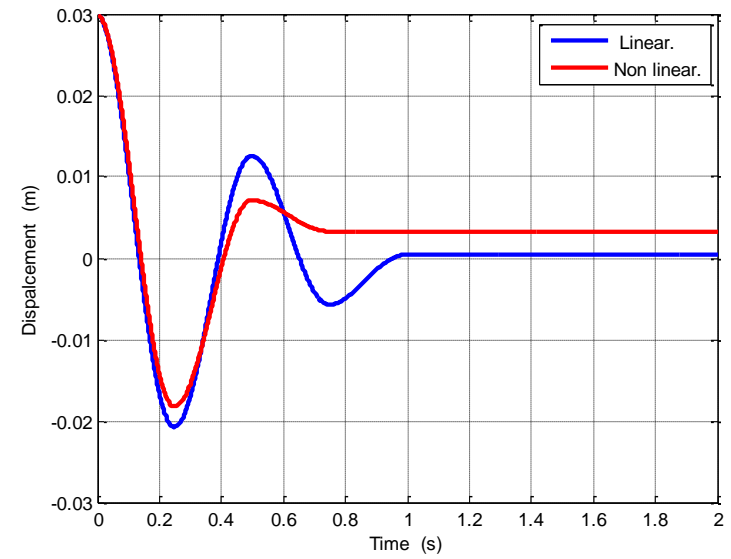

Figure 3: Linear and non-linear displacement.

\subsection{Stochastic case :}

To evaluate the variability of the dynamic response of the nonlinear damping with random parameters, a direct simulation of Monte Carlo can be used, this method is a reference for calculations, however the prohibitive calculation time limits its use. We present the perturbation method; it is based on the Taylor series development of the response around the mean values of the random variables. The parameters of equation (2) are functions of the random variables $\vartheta_{i}$. They are extended through the Taylor series of the second order as follows:

$$
\begin{gathered}
{[m]=[m]^{0}+[m]^{n} d \vartheta_{n}+[m]^{n p} d \vartheta_{n} d \vartheta_{p}} \\
{[k]=[k]^{0}+[k]^{n} d \vartheta_{n}+[k]^{n p} d \vartheta_{n} d \vartheta_{p}} \\
{[c]=[c]^{0}+[c]^{n} d \vartheta_{n}+[c]^{n p} d \vartheta_{n} d \vartheta_{p}}
\end{gathered}
$$

Where: $\mathrm{d} \vartheta_{\mathrm{i}}=\vartheta_{\mathrm{i}}-\overline{\vartheta_{1}}$ with $\overline{\vartheta_{1}}$ is the average

$[] 0,.[.]^{\mathrm{n}}$ and $[.]^{\mathrm{np}}$ are deterministic values corresponding to partial derivatives of zero, first and second order with respect to the random parameter $\vartheta_{\mathrm{i}}$. and given by:

$$
\begin{gathered}
{[\mathrm{X}]^{0}=\left.[\mathrm{X}](\vartheta)\right|_{\vartheta=\bar{\vartheta}}} \\
{[\mathrm{X}]^{\mathrm{n}}=\left.\frac{\partial[\mathrm{X}](\vartheta)}{\partial \vartheta_{\mathrm{n}}}\right|_{\vartheta=\bar{\vartheta}}} \\
{[\mathrm{X}]^{\mathrm{np}}=\left.\frac{1}{2} \frac{\partial^{2}[\mathrm{X}](\vartheta)}{\partial \vartheta_{\mathrm{n}} \partial \vartheta_{\mathrm{p}}}\right|_{\vartheta}=\bar{\vartheta}}
\end{gathered}
$$

The unknown vectors of displacement, velocity and acceleration are also developed through the Taylor series as follows:

$$
\begin{aligned}
& \{x\}=\{x\}^{0}+\{x\}^{\mathrm{n}} \mathrm{d} \vartheta_{\mathrm{n}}+\{x\}^{\mathrm{np}} \mathrm{d} \vartheta_{\mathrm{n}} \mathrm{d} \vartheta_{\mathrm{p}} \\
& \{\dot{x}\}=\{\dot{x}\}^{0}+\{\dot{x}\}^{\mathrm{n}} \mathrm{d} \vartheta_{\mathrm{n}}+\{\dot{x}\}^{\mathrm{np}} \mathrm{d} \vartheta_{\mathrm{n}} \mathrm{d} \vartheta_{\mathrm{p}} \\
& \{\ddot{x}\}=\{\ddot{x}\}^{0}+\{\ddot{x}\}^{\mathrm{n}} \mathrm{d} \vartheta_{\mathrm{n}}+\{\ddot{x}\}^{\mathrm{np}} \mathrm{d} \vartheta_{\mathrm{n}} \mathrm{d} \vartheta_{\mathrm{p}}
\end{aligned}
$$

By substituting these developments in the motion equation and writing the terms of the same order, we obtain the following differential systems:

- Equation zero order :

$$
[m]^{0}\{\ddot{x}\}^{0}+[c]^{0}\{\dot{x}\}^{0}+[k]^{0}\{x\}^{0}+\left\{f_{n l}\right\}^{0}=\left\{f_{e}\right\}
$$

- Equation first order :

$$
\begin{gathered}
{[m]^{0}\{\ddot{x}\}^{n}+[c]^{0}\{x\}^{n}+[k]^{0}\{x\}^{n}+[m]^{n}\{\ddot{x}\}^{0}+[c]^{n}\{\dot{x}\}^{0}} \\
+[k]^{n}\{x\}^{0}+\left\{f_{n l}\right\}^{n}=0
\end{gathered}
$$

- Equation second order :

$$
\begin{array}{r}
{[m]^{0}\{\ddot{x}\}^{n p}+[c]^{0}\{\dot{x}\}^{n p}+[k]^{0}\{x\}^{n p}+[m]^{n p}\{\ddot{x}\}^{0}+} \\
{[c]^{n p}\{\dot{x}\}^{0}+[k]^{n p}\{x\}^{0} \text { irst order: }+2 .[m]^{n}\{\ddot{x}\}^{p}+} \\
\text { 2. }[c]^{n}\{\dot{x}\}^{p}+2 .[k]^{n}\{x\}^{p}+\left\{f_{n l}\right\}^{n p}=0
\end{array}
$$

The temporal resolution of these equations gives the following expressions of the mean and variance of displacement $\mathrm{x}(\mathrm{t})$ :

$$
\begin{aligned}
& E(\{x(t)\})=\{x(t)\}^{0}+\frac{1}{2}\{x(t)\}^{n n} \operatorname{var}\left(\vartheta_{n}\right) \\
& \operatorname{Var}(\{x(t)\})=\operatorname{Var}\left(\vartheta_{n}\right)+ \\
& \frac{1}{4}\left(\{x(t)\}^{n p}\right)^{2} \operatorname{Var}\left(\vartheta_{n}\right) \operatorname{Var}\left(\vartheta_{p}\right)
\end{aligned}
$$

In this paper, we took the mass parameter as a Gaussian random variable with $\vartheta_{\mathrm{m}}=2.5 \%$. 
The temporal stochastic response is determined using Monte Carlo simulation and the perturbation method. The results are represented on figures. 4 and 5. They respectively represent the mean and variance of temporal displacement. It can be seen that the two stochastic analysis methods provide very similar results with a significant decrease calculation cost .

\section{Conclusion}

In this paper, we have developed Taylor's second order expansion perturbation method. It make possible to determine the stochastic response of a structure with nonlinear damping. We validated the proposed methodology for a system with one degree of freedom. We obtained similar results by the perturbation method and Monte Carlo simulation. The perturbation method reduces the calculation time sufficiently. We project an extension for a structure with several degrees of freedom and industrial structures.

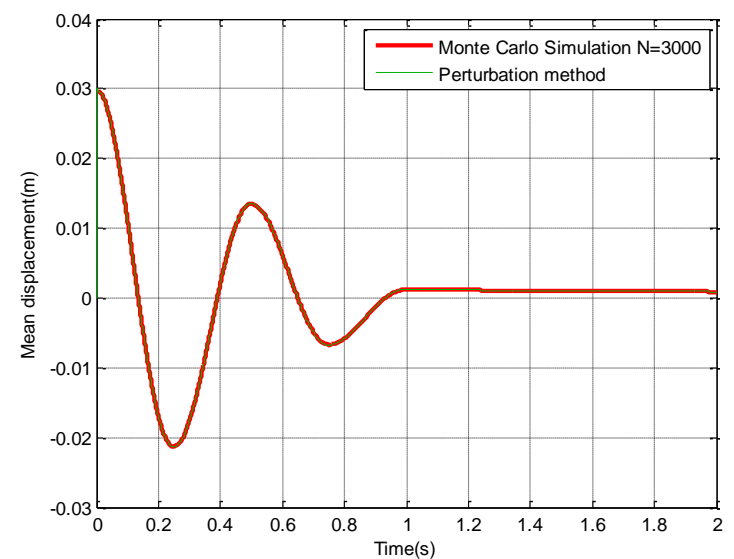

Figure 4: Mean of temporal displacement by Monte Carlo simulation $\mathrm{N}=3000$ and perturbation method.

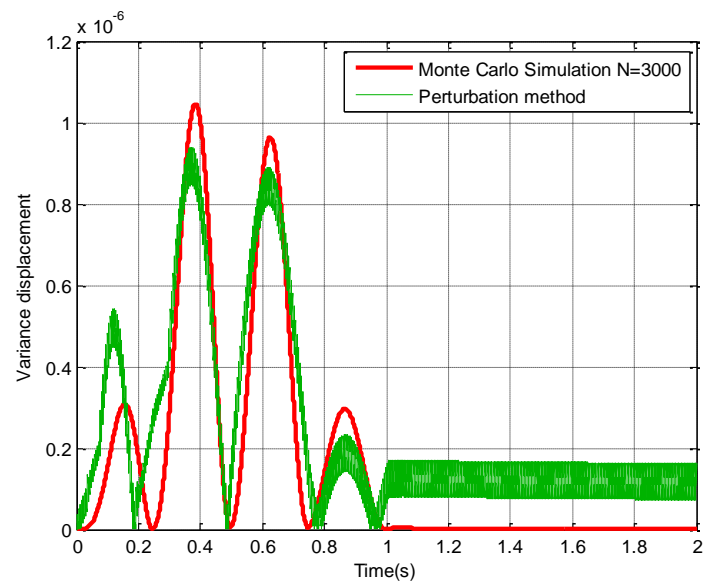

Figure 5: variance of temporal displacement by Monte Carlo simulation $\mathrm{N}=3000$ and perturbation method.

\section{References:}

[1]. J. Wallaschek, Dynamics of nonlinear automobile shock-absorbers. Int. J. Non-linear Mech. 25, (1990), 299-308.

[2] PS. Chudinov, The motion of a point mass in a medium with a square law of drag. J. Appl. Math. Mech. 65, (2001), 421-426.

[3] A. Fellowes, T. Wilson, G. Kemble, C. Havill, J. Wright. Wing box nonlinear structural damping. In Proc. 15th Int. Forum on Aeroelasticity and Structural Dynamics (IFASD 2011), Paris, France, 2011.

[4] Eichler A, Moser J, Chaste J, Zdrojek M, Wilson-Rae I, Bachtold A. Nonlinear damping in mechanical resonators made from carbon nanotubes and graphene. Nat. Nanotechnol. 6, (2011), 339-342.

[5] G.S. Fishman. Monte Carlo: Concepts, algorithms and Applications. Springer Verlag, 1996.

[6] M. Lamrhari, D. Sarsri, L. Azrar, M. Rahmoune, K. Sbai, Non linear vibrations of large structures with uncertain parameters, Advances in Mechanical Engineering, volume 9, (2017), 1-11. 